\title{
ORBIFOLD HOMEOMORPHISM AND DIFFEOMORPHISM GROUPS
}

\author{
JOSEPH E. BORZELLINO
}

VICTOR BRUNSDEN

\begin{abstract}
In this paper we outline results on orbifold diffeomorphism groups that were presented at the International Conference on Infinite Dimensional Lie Groups in Geometry and Representation Theory at Howard University, Washington DC on August 17-21, 2000. Specifically, we define the notion of reduced and unreduced orbifold diffeomorphism groups. For the reduced orbifold diffeomorphism group we state and sketch the proof of the following recognition result: Let $\mathcal{O}_{1}$ and $\mathcal{O}_{2}$ be two compact, locally smooth orbifolds. Fix $r \geq 0$. Suppose that $\Phi: \operatorname{Diff}_{\text {red }}^{r}\left(\mathcal{O}_{1}\right) \rightarrow \operatorname{Diff}_{\text {red }}^{r}\left(\mathcal{O}_{2}\right)$ is a group isomorphism. Then $\Phi$ is induced by a (topological) homeomorphism $h: X_{\mathcal{O}_{1}} \rightarrow X_{\mathcal{O}_{2}}$. That is, $\Phi(f)=h f h^{-1}$ for all $f \in \operatorname{Diff}_{\text {red }}^{r}\left(\mathcal{O}_{1}\right)$. Furthermore, if $r>0, h$ is a $C^{r}$ manifold diffeomorphism when restricted to the complement of the singular set of each stratum. We then show that if we replace the reduced orbifold diffeomorphism group by the unreduced orbifold diffeomorphism group in the above theorem, we can strengthen the homeomorphism $h$ to an orbifold homeomorphism (orbifold structure preserving). Lastly, we state a structure theorem for the orbifold diffeomorphism group, showing that it is a Banach manifold for $1 \leq r<\infty$ and a Fréchet manifold if $r=\infty$. As a corollary of this we obtain extensions of theorems of the second author to the setting of a smooth, compact orbifold.
\end{abstract}

\section{Introduction}

Orbifolds are a useful and interesting generalization of the notion of manifolds. They were first studied by Satake ${ }^{24,25}$ where they were referred to as $V$ manifolds and later by Thurston. ${ }^{27}$ In addition to being objects of study in their own right, they have come up as a new and unfamiliar domain one must pass through in order to study problems about manifolds. Some examples are the recent solution to the Arnold Conjecture by Fukaya and Ono, ${ }^{20}$ the study of convergence of Riemannian manifolds by Anderson and Cheeger, ${ }^{1}$ and their occurrence in problems in low dimensional topology, as in Scott ${ }^{26}$. One might also consider to what extent theorems about manifolds carry over 
to orbifolds. For example, many important theorems of Riemannian geometry have nice generalizations in the orbifold category. See Borzellino ${ }^{7,8,9}$ and Borzellino and Zhu. ${ }^{14}$ Of interest to us here are the appropriate orbifold versions of the recognition theorems of Filipkiewicz, ${ }^{19}$ Rubin, ${ }^{22}$ Rybicki ${ }^{23}$ for manifolds given their homeomorphism (resp. diffeomorphism) groups and the refinements of these by Banyaga to recognition theorems for symplectic, ${ }^{2,6}$ contact $^{3,4}$ and smooth unimodular ${ }^{4,5}$ structures. In all of these recognition theorems, one of the primary ingredients needed is that the group of structure preserving transformations act in some sense transitively. In trying to extend similar results to the category of orbifolds, we immediately find that this primary ingredient is missing. Orbifolds are not homogeneous objects in the sense that manifolds without boundary are. Instead, one of the distinguishing features is that they may have a nowhere dense singular set that must be preserved under any structure preserving transformation. Even though the underlying topological space of an orbifold with non-empty singular set may be a manifold, the transformations that preserve the orbifold structure will never act transitively.

Another difficulty is that many of the fundamental notions in the manifold category do not have a unique correct generalization to the category of orbifolds. Despite this, however, many manifold recognition results when formulated with proper care give the appropriate analogues in the orbifold category.

Our results may be summarized by the following theorems. The first is a partial recognition theorem for orbifold structures. While not an exact analogue for the recognition theorems mentioned above, it does give an indication of the kinds of results that can be proven and the phenomena that typically arise in the orbifold category.

Theorem A. (Borzellino and Brunsden ${ }^{10}$ ) Let $\mathcal{O}_{1}$ and $\mathcal{O}_{2}$ be two compact, locally smooth orbifolds. Fix $r \geq 0$. Suppose that $\Phi: \operatorname{Diff}_{\text {red }}^{r}\left(\mathcal{O}_{1}\right) \rightarrow$ Diff red $\left(\mathcal{O}_{2}\right)$ is a group isomorphism. Then $\Phi$ is induced by a homeomorphism $h: X_{\mathcal{O}_{1}} \rightarrow X_{\mathcal{O}_{2}}$. That is, $\Phi(f)=h f h^{-1}$ for all $f \in \operatorname{Diff}_{\text {red }}^{r}\left(\mathcal{O}_{1}\right)$. Furthermore, if $r>0, h$ is a $C^{r}$ manifold diffeomorphism when restricted to the complement of the singular set of each stratum.

Here, Diff red $(\mathcal{O})$ denotes the reduced $C^{r}$ orbifold diffeomorphism group and $X_{\mathcal{O}}$ the underlying topological space of an orbifold $\mathcal{O}$. Note that in our first paper ${ }^{10}$, all diffeomorphisms were reduced orbifold diffeomorphisms. There is also a notion of unreduced $C^{r}$ orbifold diffeomorphism group, and the corresponding result using these groups is given as Theorem B below.

The restriction to compact orbifolds cannot be removed if one insists on using the reduced orbifold maps as the following example shows. 
Example 1. Let $\mathcal{O}_{1}=(0,1)$ and $\mathcal{O}_{2}=[0,1]$, the open and closed unit intervals. These orbifolds have the same homeomorphism group, but are clearly not homeomorphic spaces.

Also, in general, the homeomorphism $h$ in Theorem $\mathrm{A}$ is not necessarily an orbifold homeomorphism and Theorem $\mathrm{A}$ is in some sense the best one can hope for if one insists on using reduced orbifold diffeomorphism groups. To see this, consider the following

Example 2. Let $\mathcal{O}_{i},(i=1,2)$ be two so-called $\mathbb{Z}_{p_{i}}$-teardrops with $p_{1} \neq p_{2}$. It is clear that the homeomorphism groups of $\mathcal{O}_{i}$ are each isomorphic to the subgroup of the homeomorphism group of the 2-sphere $S^{2}$ which fix the north pole. To see this, just observe that any homeomorphism of $S^{2}$ that fixes the north pole can be locally lifted to a $p_{i}$-fold covering of a neighborhood of the north pole. Note, however, that the orbifolds themselves are not orbifold homeomorphic, even though their underlying spaces $X_{\mathcal{O}_{i}}=S^{2}$, are topologically homeomorphic.

One might also notice that the work of Banyaga, ${ }^{4}$ Filipkiewicz, ${ }^{19}$ Rubin ${ }^{22}$ and Rybicki ${ }^{23}$ collectively show that any automorphism of the structure preserving group of transformations in the topological (with or without boundary), differentiable, PL, Lipschitz, symplectic and contact categories must be an inner automorphism. For the orbifold category, this is not the case, as the following example shows.

Example 3. (Borzellino and Brunsden ${ }^{11}$ ) For each $n>1$ there exists a compact connected orbifold $\mathcal{O}$ of dimension $n$, such that the group of automorphisms Aut $\left(\operatorname{Diff}_{\text {red }}^{r}(\mathcal{O})\right) \neq \operatorname{Inn}\left(\operatorname{Diff}_{\text {red }}^{r}(\mathcal{O})\right)$, the group of inner automorphisms. To see this, parameterize $S^{2}$ with spherical coordinates $(\theta, \phi), 0 \leq \theta<2 \pi$, $-\pi / 2 \leq \phi \leq \pi / 2$. Let $a=(\theta,-\pi / 2)$ be the north pole and $b=(\theta, \pi / 2)$ be the south pole. Give $S^{2}$ the structure of a $(p, q)$-football orbifold $\mathcal{F}$ with $p \neq q$ so the singular set $=\{a\} \cup\{b\}$. It is not hard to see that $\operatorname{Diff}_{\text {red }}^{r}(\mathcal{F})$ is isomorphic to the group of $C^{r}$ diffeomorphisms of $S^{2}$ which fix $a$ and $b$ pointwise. Consider the group automorphism $\Phi: \operatorname{Diff}_{\text {red }}^{r}(\mathcal{F}) \rightarrow \operatorname{Diff}_{\text {red }}^{r}(\mathcal{F})$ defined by $(\Phi(f))=g \circ f \circ g^{-1}$ where $g(\theta, \phi)=(\theta,-\phi)$. Then $\Phi \notin \operatorname{Inn}(\operatorname{Diff} r$ red $(\mathcal{F}))$. To see this, suppose $\Phi \in \operatorname{Inn}\left(\operatorname{Diff}_{\text {red }}^{r}(\mathcal{F})\right)$, so that there exists $h \in \operatorname{Diff}_{\text {red }}^{r}(\mathcal{F})$ with $\Phi(f)=\Psi(f)=h \circ f \circ h^{-1}$ for all $f \in \operatorname{Diff}_{\text {red }}^{r}(\mathcal{F})$. Choose a neighborhood $U_{a}$ of $a$ with $h\left(U_{a}\right) \cap g\left(U_{a}\right)=\emptyset$, and let $f_{0} \in \operatorname{Diff}_{\text {red }}^{r}(\mathcal{F})$ with $\operatorname{supp}\left(f_{0}\right) \subset U_{a}$. Then $\Phi\left(f_{0}\right)=g \circ f_{0} \circ g^{-1}$ has support in $g\left(U_{a}\right)$, a neighborhood of $b$. However, $\Psi\left(f_{0}\right)=h \circ f_{0} \circ h^{-1}$ has support in $h\left(U_{a}\right)$, a neighborhood of $a$. Thus $\Phi \neq \Psi$, and we conclude that $\Phi$ is not an inner automorphism. Higher dimensional examples can be constructed by considering products with spheres $\mathcal{F} \times S^{n}$.

Remark 4. This behavior cannot occur for one-dimensional orbifolds since the only non-trivial 1 -orbifolds are closed rays and closed intervals. The results 
in Borzellino and Brunsden ${ }^{10}$ are enough to exclude such examples since they can have only $\mathbb{Z}_{2}$ singularities.

One can, however, show that Theorem A admits a generalization which does give the analogue of the reconstruction result that holds for manifolds. To show this, we must work with a different notion of orbifold diffeomorphism, namely the unreduced orbifold diffeomorphisms.

Theorem B. Let $\mathcal{O}_{1}$ and $\mathcal{O}_{2}$ be two compact, locally smooth orbifolds. Fix $r \geq 0$. Suppose that $\Phi: \operatorname{Diff}_{\text {Orb }}^{r}\left(\mathcal{O}_{1}\right) \rightarrow \operatorname{Diff}_{\text {Orb }}^{r}\left(\mathcal{O}_{2}\right)$ is a group isomorphism. Then $\Phi$ is induced by a $C^{r}$ orbifold diffeomorphism $h: X_{\mathcal{O}_{1}} \rightarrow X_{\mathcal{O}_{2}}$. That is, $\Phi(f)=h f h^{-1}$ for all $f \in \operatorname{Diff}_{\text {Orb }}^{r}\left(\mathcal{O}_{1}\right)$.

Here, Diff ${ }_{\text {Orb }}^{r}(\mathcal{O})$ denotes the unreduced $C^{r}$ orbifold diffeomorphism group.

The above results show that the algebraic structure of the homeomorphism (resp. diffeomorphism) groups determines the orbifold (but only the topological structure of the orbifold in the case of the reduced diffeomorphism group).

A related problem to consider is determining the topological structure of the diffeomorphism group of an orbifold. In the case of a compact manifold, it is well known that the group of $C^{r}$ diffeomorphisms is a manifold for $0<r \leq$ $\infty$ where the model space is the space of $C^{r}$ tangent vector fields on $M$. See, for example Banyaga ${ }^{4}$. This is a Banach space for $0<r<\infty$ and a Fréchet space for $r=\infty$. One might naively think that the orbifold diffeomorphism group is itself an infinite dimensional orbifold, but one only need remember that the orbifold diffeomorphism group is a group and hence is homogeneous. Thus, it cannot be a non-trivial orbifold. In fact, in the case of a smooth compact orbifold, the structure of the orbifold diffeomorphism group holds no surprises.

Theorem C. Let $\mathcal{O}$ be a smooth compact orbifold without boundary and let Diff $_{\text {Orb }}^{r}(\mathcal{O})$ be the group of unreduced $C^{r}$ orbifold diffeomorphisms equipped with the topology of uniform convergence of all derivatives of orders $\leq r$. Then $\operatorname{Diff}_{\mathrm{Orb}}^{r}(\mathcal{O})$ is a manifold modeled on the topological vector space $D_{\text {Orb }}^{r}(\mathcal{O})$ of $C^{r}$ orbifold sections of the tangent orbibundle equipped with the topology of uniform convergence of derivatives of order $\leq r$. This vector space is a Banach space if $r<\infty$ and is a Fréchet space if $r=\infty$.

The rest of the paper will be devoted primarily to giving the background information and terminology used the statements of the above theorems. We will also give an indication of the proofs of Theorem A and Theorem B. The full proof of Theorem A can be found in our first paper ${ }^{10}$ and the proofs of Theorem B and Theorem $\mathrm{C}$ will appear elsewhere in ${ }^{12}$ and ${ }^{13}$. Since it is a fundamental tool in proving both Theorems A and B, we recall the following 
theorem of Rubin ${ }^{22}$. A subset $S$ of a topological space $X$ is called somewhere dense if the interior of its closure is nonempty. That is, $\operatorname{int}(\operatorname{cl}(S))=\emptyset$.

Theorem (Rubin). Let $X_{i},(i=1,2)$ be locally compact Hausdorff spaces and $G_{i}$ subgroups of the group of homeomorphisms of $X_{i}$ such that for every open set $T \subset X_{i}$ and $x \in T$ the set $\left\{g(x) \mid g \in G_{i}\right.$ and $\left.\left.g\right|_{\left(X_{i}-T\right)}=\mathrm{Id}\right\}$ is somewhere dense. Then if $\Phi: G_{1} \rightarrow G_{2}$ is a group isomorphism, then there is a homeomorphism $h$ between $X_{1}$ and $X_{2}$ such that for every $g \in G_{1}$, $\Phi(g)=h g h^{-1}$.

As a Corollary of the above results, we obtain generalizations of results of the second author (see Brunsden ${ }^{16,17}$ ) to actions of finitely generated groups on smooth compact orbifolds.

Corollary 5. Let $\mathcal{O}$ be a smooth compact orbifold, $\Gamma$ a finitely generated group and $\phi \in \operatorname{Hom}\left(\Gamma, \operatorname{Diff}_{\mathrm{Orb}}^{r}(\mathcal{O})\right)$ an action of $\Gamma$ on $\mathcal{O}$ by $C^{r}$ orbifold diffeomorphisms $(r>1)$. If $H^{1}\left(\Gamma, D_{\mathrm{Orb}}^{r-1}(\mathcal{O})\right)=0$, then there is a neighborhood $U$ of $\phi$ in $\operatorname{Hom}\left(\Gamma, \operatorname{Diff} \mathrm{Orb}^{r}(\mathcal{O})\right)$ (equipped with the compact-open topology) so that for each $\psi \in U$, there is an $h \in \operatorname{Diff}_{\text {Orb }}^{r-1}(\mathcal{O})$ so that

$$
\psi(\gamma) \circ h=h \circ \phi(\gamma)
$$

for all $\gamma \in \Gamma$. If $r=1$, then we require that in addition $H^{0}\left(\Gamma, D_{\text {Orb }}^{0}(\mathcal{O})\right)=0$. Here, $D_{\text {Orb }}^{0}(\mathcal{O})$ is as in the proof of Theorem $C$ and is a $\Gamma$ module via the induced orbibundle map $\gamma_{*}: D_{\text {Orb }}^{r}(\mathcal{O}) \rightarrow D_{\text {Orb }}^{r}(\mathcal{O})$

\section{Orbifolds}

Our definition is modeled on the definition in Thurston. ${ }^{27}$

Definition 6. A (topological) orbifold $\mathcal{O}$, consists of a paracompact, Hausdorff topological space $X_{\mathcal{O}}$ called the underlying space, with the following local structure. For each $x \in X_{\mathcal{O}}$ and neighborhood $U$ of $x$, there is a neighborhood $U_{x} \subset U$, an open set $\tilde{U}_{x} \cong \mathbb{R}^{n}$, a finite group $\Gamma_{x}$ acting continuously and effectively on $\tilde{U}_{x}$ which fixes $0 \in \tilde{U}_{x}$, and a homeomorphism $\phi_{x}: \tilde{U}_{x} / \Gamma_{x} \rightarrow U_{x}$ with $\phi_{x}(0)=x$. These actions are subject to the condition that for a neighborhood $U_{z} \subset U_{x}$ with corresponding $\tilde{U}_{z} \cong \mathbb{R}^{n}$, group $\Gamma_{z}$ and homeomorphism $\phi_{z}: \tilde{U}_{z} / \Gamma_{z} \rightarrow U_{z}$, there is an embedding $\tilde{\psi}: \tilde{U}_{z} \rightarrow \tilde{U}_{x}$ and an injective homomorphism $f: \Gamma_{z} \rightarrow \Gamma_{x}$ so that $\tilde{\psi}$ is equivariant with respect to the $f$ (that is, for $\gamma \in \Gamma_{z}, \tilde{\psi}(\gamma y)=f(\gamma) \tilde{\psi}(y)$ for all $\left.y \in \tilde{U}_{z}\right)$, such that the following diagram 
commutes:

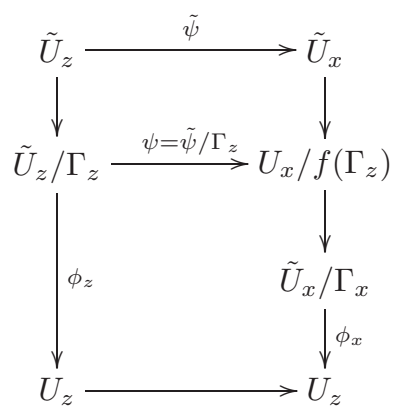

The covering $\left\{U_{x}\right\}$ of $X_{\mathcal{O}}$ is not an intrinsic part of the orbifold structure. We regard two coverings to give the same orbifold structure if they can be combined to give a larger covering still satisfying the definitions.

Let $0 \leq r \leq \infty$. An orbifold $\mathcal{O}$ is a $C^{r}$ orbifold if each $\Gamma_{x}$ acts $C^{r}$ smoothly and the embedding $\tilde{\psi}$ is $C^{r}$.

Definition 7. We say that an orbifold $\mathcal{O}$ is locally smooth if the action of $\Gamma_{x}$ on $\tilde{U}_{x} \cong \mathbb{R}^{n}$ is an orthogonal action for all $x \in \mathcal{O}$. That is, for each $x \in \mathcal{O}$, there exists a representation $L: \Gamma_{x} \rightarrow \mathbb{O}(n)$ such that if $\gamma \cdot y$ denotes the $\Gamma_{x}$ action on $\tilde{U}_{x}$, then we have $\gamma \cdot y=L(\gamma) y$ for all $y \in \tilde{U}_{x}$.

Definition 8. An orbifold chart about $x$ in a locally smooth orbifold $\mathcal{O}$ is a 4-tuple $\left(\tilde{U}_{x}, \Gamma_{x}, \rho_{x}, \phi_{x}\right)$ where $\tilde{U}_{x}=\mathbb{R}^{n}, \Gamma_{x}$ is a finite group, $\rho_{x}$ is a representation of $\Gamma_{x}: \rho_{x} \in \operatorname{Hom}\left(\Gamma_{x}, \mathbb{O}(n)\right)$, with $\mathbb{O}(n)$ the orthogonal group, and $\phi_{x}$ is a homeomorphism: $\phi_{x}: \tilde{U}_{x} / \rho_{x}\left(\Gamma_{x}\right) \rightarrow U_{x}$, where $U_{x} \subset X_{\mathcal{O}}$ is a (sufficiently small) open relatively compact neighborhood of $x$, and $\phi_{x}(0)=x$.

For convenience we will often refer to the neighborhood $U_{x}$ as an orbifold chart, and ignore the representation $\rho_{x}$ and write $U_{x}=\tilde{U}_{x} / \Gamma_{x}$. If necessary we will denote by $\pi_{x}: \tilde{U} \rightarrow \tilde{U} / \rho_{x}\left(\Gamma_{x}\right)$, the quotient map defined by the action of $\rho_{x}\left(\Gamma_{x}\right)$ on $\tilde{U}$

In the remainder, all orbifolds will be assumed to be locally smooth.

Definition 9. Let $\mathcal{O}$ be a connected $n$-dimensional locally smooth orbifold. Given a point $x \in \mathcal{O}$, there is a neighborhood $U_{x}$ of $x$ which is homeomorphic to a quotient $\tilde{U}_{x} / \Gamma_{x}$ where $\tilde{U}_{x}$ is homeomorphic to $\mathbb{R}^{n}$ and $\Gamma_{x}$ is a finite group acting orthogonally on $\mathbb{R}^{n}$. The definition of orbifold implies that the germ of this action in a neighborhood of the origin of $\mathbb{R}^{n}$ is unique. We define the isotropy group of $x$ to be the group $\Gamma_{x}$. The singular set of $\mathcal{O}$ is the set of points $x \in \mathcal{O}$ with $\Gamma_{x}=\{1\}$. Denote the singular set of $\mathcal{O}$ by $\Sigma_{1}$. Then $\Sigma_{1}$ is also a (possibly disjoint) union $\bigcup_{l_{1}} \Sigma^{\left(l_{1}\right)}$ of connected locally smooth orbifolds of strictly lower dimension (though different components may have 
different dimensions). See the section of examples. Each of the orbifolds $\Sigma_{1}^{\left(l_{1}\right)}$ has a singular set $\bigcup_{l_{2}} \Sigma_{1}^{\left(l_{1}\right)\left(l_{2}\right)}$. Define the singular set of $\Sigma_{1}$ to be $\Sigma_{2}=\bigcup_{\left(l_{1}\right)\left(l_{2}\right)} \Sigma_{1}^{\left(l_{1}\right)\left(l_{2}\right)}$. Proceeding inductively, we get a stratification of $\mathcal{O}$ :

$$
\mathcal{O}=\Sigma_{0} \supset \Sigma_{1} \supset \Sigma_{2} \supset \cdots \Sigma_{k-1} \supset \Sigma_{k}=\emptyset \text { for some } k \leq n+1
$$

By a result of M.H.A Newman ${ }^{18}$, we note that the singular set of a topological orbifold is a closed nowhere dense set. See also Thurston. ${ }^{27}$

Products of (locally smooth) orbifolds inherit a natural (locally smooth) orbifold structure:

Definition 10. Let $\mathcal{O}_{i}$ for $i=1,2$ be orbifolds. The orbifold product $\mathcal{O}_{1} \times \mathcal{O}_{2}$ is the orbifold having the following structure:

1. $X_{\mathcal{O}_{1} \times \mathcal{O}_{2}}=X_{\mathcal{O}_{1}} \times X_{\mathcal{O}_{2}}$.

2. For each $(x, y) \in X_{\mathcal{O}_{1} \times \mathcal{O}_{2}}$ and pair of orbifold charts $U_{x} \ni x$ and $V_{y} \ni y$ $U_{x} \times V_{y}$ is an orbifold chart around $(x, y)$. Explicitly,

$$
\left(\tilde{U}_{x} \times \tilde{V}_{y}, \Gamma_{x} \times \Gamma_{y}, \rho_{x} \times \rho_{y}, \phi_{x} \times \phi_{y}\right)
$$

is an orbifold chart around $(x, y)$.

Note that the isotropy group $\Gamma_{(x, y)}=\Gamma_{x} \times \Gamma_{y}$.

We close this section with some elementary results on orbifolds. The proofs can be found in our paper, ${ }^{10}$ so we omit them here.

Proposition 11. If $\mathcal{O}$ is locally smooth then in each local orbifold chart $\tilde{U}_{x}$ the fixed point set $S_{x}=\left\{y \in \tilde{U}_{x} \mid \Gamma_{x} \cdot y=y\right\}$ is a topological sub-manifold of $\tilde{U}_{x}$.

Proposition 12. If $\mathcal{O}$ is a smooth $C^{r}$ orbifold with $r>0$, then it is locally smooth.

\section{Examples of Orbifolds}

Example 13. Let $\mathcal{O}=\left(S^{n}\right.$, can $) / G, n>1$, be the $n$-dimensional hemisphere of constant curvature 1 (topologically $\mathcal{O}$ is just the closed $n$-disk $D^{n}$ ). $G=$ $\mathbb{Z}_{2} \subset \mathbb{O}(n+1)$ is the group generated by reflection through an equatorial $(n-1)$-sphere. In this case $\Sigma_{1}$ is the equatorial $(n-1)$-sphere.

Example 14. Let $\mathcal{O}$ be a $\mathbb{Z}_{p}$-football. $\mathcal{O}=\left(S^{2}\right.$, can $) / G$, where $G \subset \mathbb{O}(3)$ is rotation around the $z$-axis in $\mathbb{R}^{3}$, through an angle of $2 \pi / p$. Here $\Sigma_{1}=$ $\{$ north pole $\} \cup\{$ south pole $\}$.

Example 15. Let $\mathcal{O}$ be a $\mathbb{Z}_{p}$-football $/ G$, where $G$ is reflection in the equator of the football that does not contain the singular points. Topologically, $\mathcal{O}$ is 
$D^{2}$. Note that the singular set $\Sigma_{1}=\{$ equator $\} \cup\{$ point $\}$, thus it is possible for different components of the singular set to have different dimensions.

Example 16. Let $\mathcal{O}=\mathbb{R}^{2} / G$, where $G$ is the crystallographic group generated by reflecting an equilateral triangle or square in each of its sides to produce a tiling of $\mathbb{R}^{2}$. Then $\mathcal{O}$ is just the closed triangle or square, with singular set the boundary of the tiling region. The stratification of $\mathcal{O}$ is as follows:

$$
\begin{gathered}
\mathcal{O}=\Sigma_{0} \supset \Sigma_{1}=\{\text { the boundary of the triangle or square }\} \\
\Sigma_{2}=\{\text { the vertices }\} \supset \Sigma_{3}=\emptyset
\end{gathered}
$$

Here, $\Sigma_{1}$ is union of the closed line segments making up the boundary of the triangle or square and each of these line segments is a 1-dimensional orbifold with 2 singular points. One should observe that $\Sigma_{1}$ is not a 1 -dimensional orbifold but a union of 1-dimensional orbifolds. The lowest dimensional stratum has dimension 0 . Note that the manifold $\Sigma_{1}-\Sigma_{2}$ is a union of open line segments. If one only quotients out by the index 2 subgroup $G_{0}$ of orientation preserving elements of $G$ then $\mathcal{O}$ becomes topologically a 2 -sphere. The complement of the singular set is topologically $\mathbb{R}^{2}-\{2$ points or 3 points $\}$

Example 17. Let $\mathcal{O}$ be a $\mathbb{Z}_{p}$-teardrop. The underlying space of this orbifold is $S^{2}$ with a single conical singularity of order $p$ at the north pole.

Example 18. Consider the group $G=\mathbb{Z}_{2} \times \mathbb{Z}_{2}$ generated by rotations of $\pi$ radians about the three coordinate axes of $\mathbb{R}^{3}$. If we consider the quotient of the 2-sphere $S^{2} / G$, we get a 2-dimensional orbifold $\mathcal{O}$ whose underlying space is topologically the 2 -sphere with 3 singular points. The sin-suspension $\Sigma_{\sin } \mathcal{O}=S^{3} / \Sigma G$ is an orientable 3 -dimensional orbifold. $\Sigma G$ denotes the suspension of the action on $S^{2}$ to $S^{3}$. In this case, $\Sigma_{1}$ is the union of the 3 line segments joining the suspension points and passing through one of the singular points of $\mathcal{O} . \Sigma_{2}$ is just the two suspension points.

Example 19. Let $L_{p}=S^{3} / G$ be a 3 -dimensional lens space. Suspend the action of $G$ to an action $\Sigma G$ on the 4 -sphere $S^{4}$. Let $\mathcal{O}=S^{4} / \Sigma G$. Then the underlying space of $\mathcal{O}$ is not a manifold (or manifold with boundary).

Example 20. An $n$-dimensional smooth manifold with corners (that is, a paracompact, Hausdorff space locally modeled on $(-\infty, \infty)^{k} \times[0, \infty)^{n-k}, 0 \leq$ $k \leq n, k$ may vary from point to point, and with smooth overlaps on the charts) is an orbifold with local model $\mathbb{R}^{n} / G$ where $G=\left(\mathbb{Z}_{2}\right)^{n-k}$ and the action of $G$ is generated by reflection through the appropriate coordinate planes $x_{i_{\ell}}=0$ for $\ell=1, \ldots n-k$. The singular set $\Sigma$ is then the boundary (those points that do not have neighborhoods homeomorphic to $\mathbb{R}^{n}$ ), and the stratification given by the fixed point sets of the various subgroups of of $G$. 


\section{Orbifold Maps}

We now discuss two natural definitions of maps between orbifolds. Note that in our first paper, ${ }^{10}$ all maps discussed were reduced orbifold maps.

Definition 21. An unreduced orbifold map $\left(f, \Theta_{f, x}, \bar{f}_{x}\right)$ from $\mathcal{O}_{1}$ to $\mathcal{O}_{2}$ consists of the following:

1. A continuous map $f: X_{\mathcal{O}_{1}} \rightarrow X_{\mathcal{O}_{2}}$ of the underlying topological spaces.

2. For each $x$, a group homomorphism $\Theta_{f, x}: \Gamma_{x} \rightarrow \Gamma_{f(x)}$

3. A germ $\bar{f}_{x}$ at 0 of a $\Theta_{f, x}$ equivariant lift $\tilde{f}_{x}: \tilde{U}_{x} \rightarrow \tilde{V}_{f(x)}$ where $\left(\tilde{U}_{x}, \Gamma_{x}, \rho_{x}, \phi_{x}\right)$ is an orbifold chart about $x,\left(\tilde{V}_{f(x)}, \Gamma_{f(x)}, \rho_{f(x)}, \phi_{f(x)}\right)$ is an orbifold chart about $f(x)$, and such that the following diagram commutes:

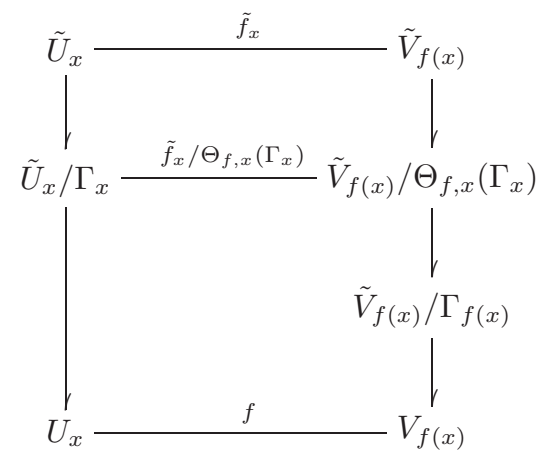

Two unreduced orbifolds maps $\left(f, \Theta_{f, x}, \bar{f}_{x}\right)$ and $\left(g, \Theta_{g, x}^{\prime}, \bar{g}_{x}\right)$ are considered the same if $f=g, \Theta_{f, x}=\Theta_{g, x}^{\prime}$, and $\bar{f}_{x}=\bar{g}_{x}$ as germs at 0 .

Definition 22. A reduced orbifold map is a continuous map $f: X_{\mathcal{O}_{1}} \rightarrow X_{\mathcal{O}_{2}}$ for which such local liftings exist. We ignore the particular choice of local lift $\tilde{f}_{x}$ and the choice of homomorphism $\Theta_{f, x}$.

Definition 23. An orbifold map $f: \mathcal{O}_{1} \rightarrow \mathcal{O}_{2}$ (either reduced or unreduced) of smooth orbifolds is $C^{r}$-smooth if each of the local lifts $\tilde{f}_{x}$ may be chosen to be $C^{r}$.

Given two orbifolds $\mathcal{O}_{i}, i=1,2$, the class of $C^{r}$ unreduced orbifold maps from $\mathcal{O}_{1}$ to $\mathcal{O}_{2}$ will be denoted by $C_{\text {Orb }}^{r}\left(\mathcal{O}_{1}, \mathcal{O}_{2}\right)$ and the class of reduced $C^{r}$ orbifold maps by $C_{\text {red }}^{r}\left(\mathcal{O}_{1}, \mathcal{O}_{2}\right)$. For the purely topological categories of locally smooth orbifolds and unreduced (respectively reduced) continuous orbifold maps we write $C_{\text {Orb }}^{0}\left(\mathcal{O}_{1}, \mathcal{O}_{2}\right)$ (respectively, $C_{\text {red }}^{0}\left(\mathcal{O}_{1}, \mathcal{O}_{2}\right)$ ). 
It is a simple matter to verify that composition of orbifold maps whether reduced or unreduced results in an orbifold map of the same type.

Definition 24. For any topological space $X$, let $H(X)$ denote its group of homeomorphisms. For a topological orbifold $\mathcal{O}$, the group of unreduced orbifold homeomorphisms, $H_{\mathrm{Orb}}(\mathcal{O})$ will be the subgroup of $H\left(X_{\mathcal{O}}\right)$ so that $f, f^{-1} \in C_{\text {Orb }}^{0}\left(X_{\mathcal{O}}, X_{\mathcal{O}}\right)$. If $\mathcal{O}$ is a $C^{r}$ orbifold, Diff ${ }_{\text {Orb }}^{r}(\mathcal{O})$ is the subgroup of $H_{\text {Orb }}(\mathcal{O})$ with $f, f^{-1} \in C_{\text {Orb }}^{r}(\mathcal{O})$. We will also use Diff Orb $(\mathcal{O})$ for $H_{\text {Orb }}(\mathcal{O})$. The corresponding notions for reduced orbifold maps will be denoted by $\operatorname{Diff}_{\text {red }}^{r}(\mathcal{O})$.

One would expect that orbifold diffeomorphisms preserve the singular set. That is, in fact, the case and we state it here for completeness. See Borzellino and Brunsden ${ }^{10}$ for the details of the proof.

Lemma 25. Any element of $\operatorname{Diff}_{\text {Orb }}^{r}(\mathcal{O})$ or $\operatorname{Diff}_{\text {red }}^{r}(\mathcal{O})$ leaves $\Sigma_{i}$ invariant (as a set), where $\Sigma_{i}$ is any substratum of $\mathcal{O}$.

The proof of Theorem A requires that apply Rubin's theorem to the complement of the singular set of an orbifold. In order to do this, we need to know that orbits of points under Diffred ${ }_{\text {red }}^{r}(\mathcal{O})$ are somewhere dense. See the previously cited paper ${ }^{10}$ for the details.

Lemma 26. The following are equivalent:

1. $x \in \mathcal{O}-\Sigma$

2. The orbit $\operatorname{Diff}_{\mathrm{red}}^{r}(\mathcal{O}) \cdot x=\left\{g(x) \mid g \in \operatorname{Diff}_{\mathrm{red}}^{r}(\mathcal{O})\right\}$ is somewhere dense.

There is an obvious forgetful homomorphism $\tau: C_{\text {Orb }}^{r}\left(\mathcal{O}_{1}, \mathcal{O}_{2}\right) \rightarrow$ $C_{\text {red }}^{r}\left(\mathcal{O}_{1}, \mathcal{O}_{2}\right)$ from the class of unreduced orbifold maps to the class of reduced orbifold maps and each reduced orbifold map comes from at least one such unreduced orbifold map.

Definition 27. For a $C^{r}$ orbifold $\mathcal{O}$, and integer $0 \leq s \leq r \leq \infty$, let $S^{s}(\mathcal{O})=$ $\operatorname{ker}\left(\tau: \operatorname{Diff}_{\text {Orb }}^{s}(\mathcal{O}) \rightarrow \operatorname{Diff}_{\text {red }}^{s}(\mathcal{O})\right)=\left\{f \in \operatorname{Diff}_{\text {Orb }}^{s}(\mathcal{O}) \mid \tau(f)=\operatorname{Id}_{\mathcal{O}}\right\}$. Since $S^{s}(\mathcal{O})$ consists of those unreduced lifts of the (always) $C^{r}$ smooth identity map, we see that $S^{s}(\mathcal{O})$ is independent of $s$, and thus we denote it more simply by $S(\mathcal{O})=S^{s}(\mathcal{O})=S^{r}(\mathcal{O})$, for all $0 \leq s \leq r \leq \infty$.

The following result gives the structure of $S(\mathcal{O})$, and its proof basically follows from the definitions. A full proof of this result is in preprint ${ }^{13}$ form.

Proposition 28. Let $x \in \mathcal{O}$, and let $\left(\tilde{U}_{x}, \Gamma_{x}, \rho_{x}, \phi_{x}\right)$ be an orbifold chart around $x$. If $\sigma=\left(\mathrm{Id}, \Theta_{\mathrm{Id}, x}, \overline{\mathrm{Id}}\right) \in S(\mathcal{O})$, then there is a $\delta \in \Gamma_{x}$ so that $\overline{\operatorname{Id}}(\tilde{y})=\delta \cdot \tilde{y}$ for all $\tilde{y} \in \tilde{U}_{x}$ and $\Theta_{\mathrm{Id}, x}(\gamma)=\delta \cdot \gamma \cdot \delta^{-1}$. Note that if $x \in \mathcal{O}$ is non-singular, then $\delta=e$. 


\section{$5 \quad$ Orbifold Bundles, Orbibundles and Suborbifolds}

Since we will ultimately want to look at sections of the tangent bundle to a smooth orbifold in order to state Theorem C, we now define the notions of unreduced and reduced orbifold bundles.

Definition 29. An unreduced (respectively, reduced) orbifold bundle is a triple $(\mathcal{E}, \mathcal{B}, p)$ where $\mathcal{E}$ and $\mathcal{B}$ are locally smooth orbifolds with $p: \mathcal{E} \rightarrow \mathcal{B}$ an unreduced (respectively, reduced) orbifold map. An orbifold bundle is linear if the orbifold structures on the total space $\mathcal{E}$ and base space $\mathcal{B}$ are compatible with the following local triviality conditions

1. For each $x \in X_{\mathcal{B}}$ with isotropy group $\Gamma_{x}$ and orbifold chart $U_{x} \subset X_{\mathcal{B}}$ containing $x$, so that $\tilde{U}_{x} \cong \mathbb{R}^{n}$ and $\tilde{U}_{x} / \Gamma_{x} \cong U_{x}$ we have $\tilde{p}^{-1}\left(\tilde{U}_{x}\right) \cong$ $\tilde{U}_{x} \times \mathbb{R}^{k}$. Also there is a group $G_{x}$, an action $P_{x} \in \operatorname{Hom}\left(G_{x}, \mathbb{O}(n+k)\right)$ and a surjective group homomorphism $\Theta_{p,(x, 0)}: G_{x} \rightarrow \Gamma_{x}$ so that $\tilde{p}$ is $\Theta_{p,(x, 0)}$ equivariant, i.e.:

$$
\rho_{x}\left(\Theta_{p,(x, 0)}(g)\right) \tilde{p}(y)=\tilde{p}\left(P_{x}(g) y\right)
$$

for all $g \in G_{x}$ and $y \in \tilde{p}^{-1}\left(\tilde{U}_{x}\right)$.

2. Given another $U_{z} \subset U_{x}$ with corresponding $\tilde{U}_{z} \cong \mathbb{R}^{n}$, group $\Gamma_{z}$, homeomorphism $\phi_{z}: \tilde{U}_{z} / \Gamma_{z} \rightarrow U_{z}$ and embedding $\tilde{\psi}: \tilde{U}_{z} \hookrightarrow \tilde{U}_{x}$ there are injective group homomorphisms $\theta_{z, x}: \Gamma_{z} \rightarrow \Gamma_{x}, \Theta_{z, x}: G_{z} \rightarrow G_{x}$ and embeddings $\tilde{\Psi}: \tilde{U}_{z} \times \mathbb{R}^{k} \rightarrow \tilde{U}_{x} \times \mathbb{R}^{k}$ and $\Psi: p^{-1}\left(U_{z}\right) \hookrightarrow p^{-1}\left(U_{x}\right)$ so that the following diagram commutes. (Note that all the vertical arrows are quotient maps obtained by mod-ding out by the action of the appropriate 
isotropy groups).

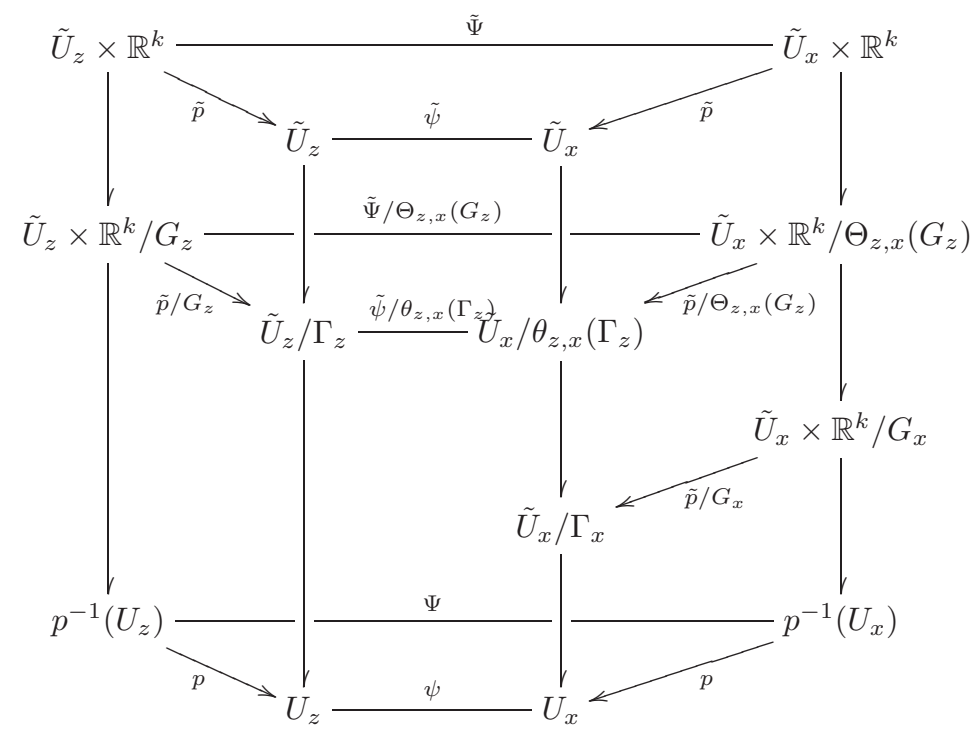

3. The mappings $\tilde{\Psi}(y, \cdot): \tilde{p}^{-1}(y) \rightarrow \tilde{p}^{-1}(\tilde{\psi}(y))$ are invertible linear maps on $\tilde{p}^{-1}(y)$ for each $y \in \tilde{U}_{z}$.

In most cases the actions $P_{x}$ above will be in $\mathbb{O}(n) \times \mathbb{O}(k)$ rather than the more general case of $\mathbb{O}(n+k)$ that we allow above.

Definition 30. A linear orbibundle is a linear orbifold bundle with the property that for all $x \in \mathcal{O}$ one has $G_{x} \cong \Gamma_{x}$.

Suppose that one is given a topological space $X_{\mathcal{E}}$, a base orbifold $\mathcal{B}$, and a map $p: X_{\mathcal{E}} \rightarrow \mathcal{B}$ satisfying conditions (1)-(3). Then $X_{\mathcal{E}}$ can be given the structure as the total space of a linear orbifold bundle $p: \mathcal{E} \rightarrow \mathcal{B}$. In particular, the total space $\mathcal{E}$ is an orbifold.

Definition 31. Let $\mathcal{O}$ be an $n$-dimensional smooth orbifold. The tangent orbibundle of $\mathcal{O}, p: T \mathcal{O} \rightarrow \mathcal{O}$ is the linear orbibundle defined by the following construction. If $U_{x}$ is an orbifold chart around $x \in \mathcal{O}, \Gamma_{x}$ the isotropy group of $x$, and $\tilde{U}_{x} \cong \mathbb{R}^{n}$ so that $U_{x} \cong \tilde{U}_{x} / \Gamma_{x}$, then $p^{-1}\left(U_{x}\right) \cong\left(\tilde{U}_{x} \times \mathbb{R}^{n}\right) / \Gamma_{x}$ where $\Gamma_{x}$ acts on $\tilde{U}_{x} \times \mathbb{R}^{n}$ via

$$
\gamma \cdot(x, v)=\left(\gamma \cdot x, d \gamma_{x}(v)\right)
$$


The definition of the tangent orbibundle allows the following

Definition 32. For a $C^{r}$ orbifold map $f: \mathcal{O}_{1} \rightarrow \mathcal{O}_{2}$, the tangent mapping Tf : $T \mathcal{O}_{1} \rightarrow T \mathcal{O}_{2}$ is the $C^{r-1}$ orbifold map defined by:

$$
\widetilde{T f}(x, v)=\left[\left(\tilde{f}(\tilde{x}), d \tilde{f}_{\tilde{x}}(\tilde{v})\right]\right.
$$

where $[(\tilde{x}, \tilde{v})]$ is the equivalence class of $(\tilde{x}, \tilde{v}) \in \tilde{U}_{x} \times \mathbb{R}^{n}$ and $(x, v) \cong[(\tilde{x}, \tilde{v})]$. One may similarly define the unreduced orbifold tangent mapping of an unreduced orbifold map by including the homomorphism information. $T f$ is an orbifold map in the sense of Definition 21.

Definition 33. An unreduced orbisection of an orbifold bundle $\mathcal{E}$ over an orbifold $\mathcal{O}$ is an unreduced orbifold map $f: \mathcal{O} \rightarrow \mathcal{E}$ such that $p \circ f=\operatorname{Id}_{\mathcal{O}}$. In other words, it is simply a section in the category of orbibundles and unreduced orbifold maps. Likewise, we may define a reduced orbisection of a reduced orbibundle by using reduced orbifold maps.

We have the following structure result for orbisections. We defer the proof to our preprint. 13

Proposition 34. The set $C_{\mathrm{Orb}}^{0}(\mathcal{E})$ of unreduced orbisections of a linear orbibundle $\mathcal{E}$ is naturally a real vector space.

Example 35. Given an orbifold $\mathcal{O}$, the orbifold product $\mathcal{O} \times \mathcal{O}$ is itself an orbifold. The projections $p_{1}$ and $p_{2}$ onto the first and second factors respectively give two different orbifold bundle structures to $\mathcal{O} \times \mathcal{O}$. Note that this bundle has a canonical orbifold section, the diagonal $=\Delta(\mathcal{O}) \subset \mathcal{O} \times \mathcal{O}$, where $\Delta: \mathcal{O} \rightarrow \mathcal{O} \times \mathcal{O}$ is defined by the diagonal map $\Delta(x)=(x, x)$ for all $x \in \mathcal{O}$ and $\Theta_{\Delta, x}(\gamma)=\gamma \times \gamma$ for all $\gamma \in \Gamma_{x}$.

We now consider suborbifolds. The definition of a suborbifold is somewhat more delicate than the corresponding notion for a manifold. We want a definition that is sufficiently flexible so that, in particular, the diagonal $\Delta(\mathcal{O}) \subset \mathcal{O} \times \mathcal{O}$ is a suborbifold of $\mathcal{O} \times \mathcal{O}$.

Definition 36. A suborbifold $\mathcal{P}$ of an orbifold $\mathcal{O}$ consists of the following.

1. A subspace $X_{\mathcal{P}} \subset X_{\mathcal{O}}$ equipped with the subspace topology

2. For each $x \in X_{\mathcal{P}}$ and neighborhood $W$ of $x$ in $X_{\mathcal{P}}$ there is an orbifold chart $\left(\tilde{U}_{x}, \Gamma_{x}, \rho_{x}, \phi_{x}\right)$ about $x$ in $\mathcal{O}$ with $U_{x} \subset W$, a subgroup $G_{x} \subset \Gamma_{x}$ of the isotropy group of $x$ in $\mathcal{O}$ and a $\rho_{x}\left(G_{x}\right)$ invariant vector subspace $\tilde{V}_{x} \subset \tilde{U}_{x}=\mathbb{R}^{n}$, so that

$$
\left(\tilde{V}_{x}, G_{x},\left.\rho_{x}\right|_{G_{x}}, \psi_{x}\right)
$$

is an orbifold chart for $\mathcal{P}$ and 
3.

$$
\begin{aligned}
V_{x} & =\psi_{x}\left(\tilde{V}_{x} / \rho_{x}\left(G_{x}\right)\right) \\
& =U_{x} \cap X_{\mathcal{P}} \\
& =\phi_{x}\left(\pi_{x}\left(\tilde{V}_{x}\right)\right)
\end{aligned}
$$

is an orbifold chart for $x$ in $\mathcal{P}$ where $\pi_{x}: \tilde{U}_{x} \rightarrow \tilde{U}_{x} / \rho_{x}\left(\Gamma_{x}\right)$ is the quotient map.

Remark 3\%. If one only requires (1) and (2) for a suborbifold $\mathcal{P}$, then we will say that the suborbifold $\mathcal{P}$ is immersed.

Remark 38. It is tempting to define the notion of an $m$-suborbifold $\mathcal{P}$ of an $n$-orbifold $\mathcal{O}$ simply by requiring $\mathcal{P}$ to be locally modeled on $\mathbb{R}^{m} \subset \mathbb{R}^{n}$ modulo finite groups. That is, the local action on $\mathbb{R}^{m}$ is induced by the local action on $\mathbb{R}^{n}$. See Thurston ${ }^{27}$ for a definition that takes this approach. This is equivalent to the added condition in our definition that $G_{x}=\Gamma_{x}$ at all $x$ in the underlying topological space of $\mathcal{P}$. By analogy with the definition of neat submanifolds of manifolds with boundary (see for example Hirsch ${ }^{21}$ ), we call such suborbifolds neat. Neat suborbifolds turn out to be too restrictive of a notion for our purposes. For example the diagonal $\Delta_{\mathcal{O}} \subset \mathcal{O} \times \mathcal{O}$ is not a neat suborbifold of $\mathcal{O} \times \mathcal{O}$. However, using the more general definition 36, the diagonal is a suborbifold.

Remark 39. Suborbifolds are therefore orbifolds whose orbifold structure is a "substructure" of the ambient orbifold $\mathcal{O}$, in the sense that the restriction to $\mathcal{P}$ of an orbifold map from $\mathcal{O}$ are themselves orbifold maps from $\mathcal{P}$.

Remark 40. Let $\mathcal{P} \subset \mathcal{O}$ be a suborbifold. Note that even though a point $p \in X_{\mathcal{P}}$ may be in the singular set of $\mathcal{O}$, it need not be in the singular set of $\mathcal{P}$.

Example 41. Let $\mathcal{O}$ be an orbifold and $\mathcal{O} \times \mathcal{O}$ be the orbifold product of $\mathcal{O}$ with itself. Let $\Delta: \mathcal{O} \rightarrow \mathcal{O} \times \mathcal{O}$ be the diagonal mapping. By construction, the diagonal $=\Delta(\mathcal{O}) \subset \mathcal{O} \times \mathcal{O}$ is a suborbifold of $\mathcal{O} \times \mathcal{O}$ with isotropy group $\Gamma_{(x, x)} \cong \Gamma_{x}$ via the diagonal action $\gamma \cdot(\tilde{x}, \tilde{x})=(\gamma \cdot \tilde{x}, \gamma \cdot \tilde{x})$.

Example 42. Let $f \in \operatorname{Diff}_{\text {Orb }}^{r}(\mathcal{O})$, then the graph of $f, \operatorname{graph}(f)$ defined by

$$
\operatorname{graph}(f)=\{(x, y) \in \mathcal{O} \times \mathcal{O} \mid y=f(x)\}
$$

in $\mathcal{O} \times \mathcal{O}$ is a suborbifold of $\mathcal{O}$. Note the isotropy group $\Gamma_{(x, y)} \cong \Gamma_{x}$ acting via the twisted diagonal action $\gamma \cdot(\tilde{x}, \tilde{y})=\left(\gamma \cdot \tilde{x}, \Theta_{f, x}(\gamma) \cdot \tilde{y}\right)$. Similarly given an orbisection $\sigma$ of an orbibundle $\mathcal{P} \rightarrow \mathcal{O}$, this defines a suborbifold of the total space of the orbibundle $\mathcal{P}$.

Definition 43. Let $\mathcal{P}$ be an $m$-dimensional $C^{r}$ suborbifold of an $n$ dimensional $C^{r}$ orbifold $\mathcal{O}$ (where $r \geq 1$ ). The normal orbibundle $N \mathcal{P}$ of 
$\mathcal{P}$ in $\mathcal{O}$ is the linear orbibundle over $\mathcal{P}$ with projection $p: N \mathcal{P} \rightarrow \mathcal{P}$ so that if $U$ is an orbifold chart in $\mathcal{P}$ about $x \in \mathcal{P}$ then

$$
\tilde{p}^{-1}(\tilde{U})=\tilde{U} \times\left(\mathbb{R}^{n} / \mathbb{R}^{m}\right)
$$

with the $\Gamma_{x}$ action defined by

$$
\gamma \cdot\left(x, v / \mathbb{R}^{m}\right)=\left(\gamma \cdot x, d \gamma_{x}\left(v / \mathbb{R}^{m}\right)\right) .
$$

\section{Extending Orbifold Diffeomorphisms and Local Contractions}

For any subgroup $G$ of the homeomorphism group $H(X)$ of a topological space $X$, let $G_{c} \subset G$ denote those elements of $G$ with compact support in $X$. Let $G_{0}$ be the subgroup of $G_{c}$ whose elements are isotopic to the identity through elements of $G$ with compactly supported isotopy. For any self-map $f: X \rightarrow X$ of a topological space $X$, let the $\operatorname{support} \operatorname{supp}(f)=\operatorname{cl}\{x \in X \mid f(x)=x\}$ where $\operatorname{cl}(S)$ denotes the closure of the set $S$. By compactly supported isotopy we mean an isotopy $f:[0,1] \times X$, $\operatorname{such}$ that $\operatorname{supp}(f) \subset[0,1] \times X$ is compact. The proofs of the following results can be found in Borzellino and Brunsden ${ }^{10}$. Proposition 44. Let $\Sigma$ denote the singular set of an orbifold $\mathcal{O}$. The group $\operatorname{Diff}^{0}(\mathcal{O}-\Sigma)_{c}$ is a subgroup of $\operatorname{Diff}_{\text {red }}^{0}(\mathcal{O})$ for any topological orbifold $\mathcal{O}$. Moreover, if $\mathcal{O}$ is $C^{r}$-smooth, then for each component $\mathcal{A}=\Sigma_{m}^{\left(l_{1}\right)\left(l_{2}\right) \cdots\left(l_{m}\right)}$ of $\Sigma_{m}$ and $f \in \operatorname{Diff}^{r}\left(\mathcal{A}-\Sigma_{\mathcal{A}}\right)_{0}$, there is an extension $g \in \operatorname{Diff}_{\text {red }}^{0}(\mathcal{O})$ and a neighborhood $U$ of $\Sigma_{m}^{\left(l_{1}\right)\left(l_{2}\right) \cdots\left(l_{m}\right)}$ in $\mathcal{O}$ such that $\operatorname{supp}(g) \subset U$ and the restriction

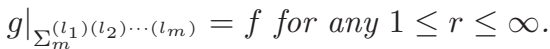

The proof Theorems A and Theorem B will require that there are enough local orbifold diffeomorphisms whose behavior under the group isomorphism can be controlled. To this end, we use the following

Definition 45. For a locally compact Hausdorff space $X$, a subgroup $G \subset$ $H(X)$ and $x \in X$, we say that $g_{x} \in G$ is a local contraction about $x$ if:

1. $x \in \operatorname{supp}\left(g_{x}\right)$ and $\operatorname{supp}\left(g_{x}\right)$ is compact

2. for all open neighborhoods $V$ and $W$ of $x$ in $\operatorname{supp}\left(g_{x}\right)$ with $\bar{W} \subset V \subset$ $\bar{V} \subset \operatorname{int}\left(\operatorname{supp}\left(g_{x}\right)\right)$ there is an $N \in \mathbb{N}$ so that $g_{x}^{n}(\bar{V}) \subset W$ for all $n>N$.

3. $g_{x}(x)=x$

For locally smooth orbifolds there are plenty of local contractions:

Proposition 46. If $\mathcal{O}$ is locally smooth, then for each $x \in \mathcal{O}$ and neighborhood $U$ of $x$ there is a (reduced) local contraction about $x$ with support in $U$. 


\section{Proof of Theorem A}

We sketch an outline of the proof of Theorem A in the locally smooth case. The full proof, including the extension to the smooth case may be found in Borzellino and Brunsden ${ }^{10}$. Note that for any open subset $U$ of an orbifold $\mathcal{O}, x \in U$ if and only if there is a neighborhood $V$ of $x$ so that $V-\Sigma \subset U-\Sigma$ and for an open subset $U$ as above, $x \in \operatorname{cl}(U)$ if and only if $(V-\Sigma) \cap(U-\Sigma)$ $=\emptyset$ for all neighborhoods $V$ of $x$. Note that these follow almost trivially from the nowhere density of the singular set. In brief, the outline of the proof is as follows.

Let $\mathcal{O}_{1}$ and $\mathcal{O}_{2}$ be two compact, locally smooth orbifolds and let $\Phi$ : $\operatorname{Diff}_{\text {red }}^{r}\left(\mathcal{O}_{1}\right) \rightarrow \operatorname{Diff}_{\text {red }}^{r}\left(\mathcal{O}_{2}\right)$ be a group isomorphism. By Lemmas 25 and 26, Proposition 44 and Rubin's theorem we have a homeomorphism $h: \mathcal{O}_{1}-\Sigma_{1} \rightarrow$ $\mathcal{O}_{2}-\Sigma_{2}$ such that for every $f \in \operatorname{Diff}_{\text {red }}^{r}\left(\mathcal{O}_{1}\right)$ we have $\Phi(f)=h f h^{-1}$. Note that this implies that the singular sets of $\mathcal{O}_{i}$ are either both empty or are both nonempty. To see this, suppose $\Sigma_{1}=\emptyset$ and that $\Sigma_{2}=\emptyset$. Then $\mathcal{O}_{2}=\mathcal{O}_{2}-\Sigma_{2}$ is a closed manifold. $\mathcal{O}_{1}-\Sigma_{1}$, however, is a non-compact manifold, and this contradicts the existence of a homeomorphism $h: \mathcal{O}_{1}-\Sigma_{1} \rightarrow \mathcal{O}_{2}-\Sigma_{2}$ guaranteed by Rubin's theorem. Since Rubin's theorem implies Theorem A when $\Sigma_{1}=\Sigma_{2}=\emptyset$ (the manifold case), we need only concern ourselves with case when $\Sigma_{1}$ and $\Sigma_{2}$ are non-empty.

Next, we extend $h$ to a bijection $\bar{h}: \mathcal{O}_{1} \rightarrow \mathcal{O}_{2}$ inducing the group isomorphism as follows:

Let $x \in \Sigma_{1}$, and let $U_{x}$ be a relatively compact open neighborhood of $x$ in $\mathcal{O}_{1}$. By Proposition 46, there exists a $g_{x} \in \operatorname{Diff}_{\text {red }}^{r}(\mathcal{O})$ which is a local contraction about $x$ with support in $U_{x}$. Let $\hat{g}_{x}=\Phi\left(g_{x}\right)$, and $\hat{U}_{x}=$ $\operatorname{int}\left(\operatorname{cl}\left(h\left(U_{x}-\Sigma_{1}\right)\right)\right.$. It follows from Rubin's theorem that $\operatorname{supp}\left(\hat{g}_{x}\right) \subset \operatorname{cl}\left(\hat{U}_{x}\right)$. For details, see our paper. ${ }^{10}$

We now show that $\hat{g}_{x}$ possesses a non-empty invariant set $Y_{x} \subset$ $\operatorname{int}\left(\operatorname{supp}\left(\hat{g}_{x}\right)\right) \cap \Sigma_{2}$. For this, Let $\hat{W} \subset \hat{U}_{x}$ be any relatively compact open subset of $\mathcal{O}_{2}$ with

$$
x \in \operatorname{int}\left(\operatorname{cl}\left(h^{-1}\left(\hat{W}-\Sigma_{2}\right)\right)\right.
$$

Compactness of $\mathcal{O}_{2}$ makes this possible. For any neighborhood $V$ of $x$ with $\operatorname{cl}(V)-\Sigma_{1} \subset h^{-1}\left(\hat{W}-\Sigma_{2}\right)$ there is an $m>0$ so that

$$
g_{x}^{m}\left(h ^ { - 1 } ( \hat { W } - \Sigma _ { 2 } ) \subset V \subset \operatorname { i n t } \left(\operatorname{cl}\left(h^{-1}\left(\hat{W}-\Sigma_{2}\right)\right)\right.\right.
$$

since $g_{x}$ is a local contraction about $x$. Therefore,

$$
x \in \bigcap_{n<N} g_{x}^{m n}\left(\operatorname{cl}\left(h^{-1}\left(\hat{W}-\Sigma_{2}\right)\right)=\emptyset\right.
$$


which implies

$$
\begin{aligned}
& \operatorname{cl} g_{x}^{m n}\left(h^{-1}\left(\hat{W}-\Sigma_{2}\right)=\emptyset\right. \\
& n<N
\end{aligned}
$$

and so by definition of $\hat{g}_{x}$ and $h$,

$$
\operatorname{cl} h^{-1}\left(\hat{g}_{x}^{m n}\left(\hat{W}-\Sigma_{2}\right)=\emptyset\right.
$$

which in turn implies,

$$
h^{-1}\left(\hat{g}_{x}^{m n}(\hat{W})-\Sigma_{2}=\emptyset\right.
$$

It now follows that

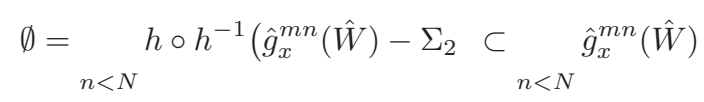

and so

$$
\hat{g}_{n<N}^{m n}(\operatorname{cl}(\hat{W}))=\emptyset
$$

Then the collection of closed sets $\left\{\hat{g}_{x}^{m n}(\operatorname{cl}(\hat{W}))\right\}$ has the finite intersection property, and so by compactness of $\mathcal{O}_{2}$ we have

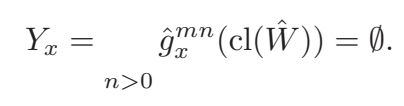

By construction, $Y_{x}=\bigcap_{m>0} \hat{g}_{x}^{m n}(\mathrm{cl}(\hat{W}))$ is a compact, $\hat{g}_{x}$ invariant set. We claim that $Y_{x}$ is independent of $g_{x}$ and the subset $\hat{W}$. To see this, suppose that $g_{x}$ is another local contraction with fixed point $x$, and $\hat{W} \subset \mathcal{O}_{2}$ is a compact subset of int $\left(\operatorname{supp}\left(\Phi\left(g_{x}\right)\right)\right.$ satisfying the same requirement of $\hat{W}$ as above. As both $g_{x}$ and $g_{x}$ are local contractions, for any $n>0$ there is an $m>0$ so that:

$$
g_{x}^{m}\left(\operatorname { i n t } ( \operatorname { c l } ( h ^ { - 1 } ( \hat { W } - \Sigma _ { 2 } ) ) ) \subset g _ { x } ^ { n } \left(\operatorname{int}\left(\operatorname{cl}\left(h^{-1}\left(\hat{W}-\Sigma_{2}\right)\right)\right)\right.\right.
$$

and for any $m>0$ there is an $n>0$ so that:

$$
g_{x}^{n}\left(\operatorname { i n t } ( \operatorname { c l } ( h ^ { - 1 } ( \hat { W } - \Sigma _ { 2 } ) ) ) \subset g _ { x } ^ { m } \left(\operatorname{int}\left(\operatorname{cl}\left(h^{-1}\left(\hat{W}-\Sigma_{2}\right)\right)\right)\right.\right.
$$

Therefore $\bigcap_{n>0} \hat{g}_{x}^{n}(\hat{W}) \subset \bigcap_{m>0} \hat{g}_{x}^{m}(\hat{W}) \subset \bigcap_{n>0} \hat{g}_{x}^{n}(\hat{W})$ which shows the independence of $Y_{x}$ on the local contraction.

Let $g_{x}$ and $g_{x^{\prime}}$ be local contractions about $x$ and $x$ respectively with disjoint supports such that $\operatorname{supp}\left(g_{x}\right) \subset U$ and $\operatorname{supp}\left(g_{x^{\prime}}\right) \subset U$ where $U$ and $U$ 
are open sets with $U \cap U=\emptyset, U=\operatorname{int}(\operatorname{cl}(U))$ and $U=\operatorname{int}(\operatorname{cl}(U))$. Therefore $h\left(U-\Sigma_{1}\right) \cap h\left(U-\Sigma_{1}\right)=\emptyset$ and by the remark above, if $z \in \operatorname{int}\left(\operatorname{cl}\left(h\left(U-\Sigma_{1}\right)\right)\right.$, then there is a neighborhood $V$ of $z$ so that $V-\Sigma_{2} \subset \operatorname{int}\left(\operatorname{cl}\left(h\left(U-\Sigma_{1}\right)\right)-\Sigma_{2}=\right.$ $h\left(U-\Sigma_{1}\right)$. Therefore $z \notin \operatorname{int}\left(\operatorname{cl}\left(h\left(U-\Sigma_{1}\right)\right)\right.$. Reversing the roles of $U$ and $U$ shows that

$$
\operatorname{int}\left(\operatorname { c l } ( h ( U - \Sigma _ { 1 } ) ) \quad \operatorname { i n t } \left(\operatorname{cl}\left(h\left(U-\Sigma_{1}\right)\right)=\emptyset\right.\right.
$$

Since $Y_{x} \subset \operatorname{int}\left(\operatorname{cl}\left(h\left(U-\Sigma_{1}\right)\right)\right.$ and $Y_{x^{\prime}} \subset \operatorname{int}\left(\operatorname{cl}\left(h\left(U-\Sigma_{1}\right)\right), Y_{x} \cap Y_{x^{\prime}}=\emptyset\right.$. Therefore for any two such subsets $Y_{x}$ and $Y_{x^{\prime}}$ of $\mathcal{O}_{2}$, if $Y_{x} \cap Y_{x^{\prime}}=\emptyset$ then $Y_{x}=Y_{x^{\prime}}$ and $x=x$.

Given a $k \in \operatorname{Diff}_{\text {red }}^{r}\left(\mathcal{O}_{1}\right), x \in \Sigma_{1}$ and a local contraction $g_{x}$ about $x$, the orbifold diffeomorphism $k \circ g_{x} \circ k^{-1}$ is a local contraction about $k(x)$. Hence $\Phi\left(k \circ g_{x} \circ k^{-1}\right)$ will have invariant set $Y_{k(x)}$. Since $\Phi$ is a group isomorphism between Diff red ${ }_{\text {re }}\left(\mathcal{O}_{1}\right)$ and Diff red $r\left(\mathcal{O}_{2}\right)$, the invariant set of $\Phi\left(k \circ g_{x} \circ k^{-1}\right)$ will be $\Phi(k)\left(Y_{x}\right)$. Therefore $\Phi(k)\left(Y_{x}\right)=Y_{k(x)}$ for all $x \in \Sigma_{1}$. We will use this below to prove that the sets $Y_{x}$ consist of a single point.

Let $y \in Y_{x}$, and $\hat{g}_{y} \in \operatorname{Diff}_{\text {red }}^{r}\left(\mathcal{O}_{2}\right)$ be a local contraction about $y$. Let $g_{y}=\Phi^{-1}\left(\hat{g}_{y}\right)$ and then by definition $y \in \hat{g}_{y}^{n}\left(Y_{x}\right)=Y_{g_{y}^{n}(x)}$ for all $n \geq 0$. Hence $Y_{x} \cap \hat{g}_{y}^{n}\left(Y_{x}\right)=\emptyset$ for all $n \geq 0$ and so $Y_{x}=\hat{g}_{y}^{n}\left(Y_{x}\right)$ for all $n \geq 0$. If $z \in Y_{x} \cap \operatorname{supp}\left(\hat{g}_{y}\right)$ then for any neighborhood $V$ of $y$ in $\mathcal{O}_{2}$, there is an $n>0$ so that $\hat{g}_{y}^{n}(z) \in V$ which implies that $Y_{x} \cap \operatorname{int}\left(\operatorname{supp}\left(\hat{g}_{y}\right)\right)=\{y\}$. Since $\hat{g}_{y}$ was essentially arbitrary, this implies that $Y_{x}=\{y\}$, that is, the invariant set $Y_{x}$ of $g_{x}$ consists of a single point.

Define the extension $\bar{h}$ of $h$ to all of $\mathcal{O}_{1}$ by the following:

$$
\bar{h}(x)= \begin{cases}h(x), & \text { if } x \in \mathcal{O}_{1}-\Sigma_{1} \\ Y_{x}, & \text { if } x \in \Sigma_{1}\end{cases}
$$

By construction, $\bar{h}$ is an injection inducing the group isomorphism. Similarly we can construct an injection $\overline{h^{-1}}$. Continuity of $\bar{h}$ follows from the following. Given $x \in \mathcal{O}_{1}$ and a neighborhood $U_{x}$ of $x$, then there is a local contraction $g_{x}$ about $x$ with support in $U_{x}$ (by Proposition 46). By construction, $x \in$ $\operatorname{int}\left(\operatorname{supp}\left(g_{x}\right)\right)$ and so the collection

$$
\mathcal{B}=\bigcup_{x \in \mathcal{O}_{1}} \bigcup_{U_{x} \ni x} \operatorname{int}\left(\operatorname{supp}\left(g_{x}\right)\right) \mid \operatorname{int}\left(\operatorname{supp}\left(g_{x}\right)\right) \subset U_{x}
$$

forms a base for the topology of $\mathcal{O}_{1}$. Let $\operatorname{Fix}(f)=\{x \in \mathcal{O} \mid f(x)=x\}$. Thus

$$
\begin{gathered}
\bar{h}\left(\left(\mathcal{O}_{1}-\operatorname{int}\left(\operatorname{supp}\left(g_{x}\right)\right)\right) \cup\{x\}=\bar{h}\left(\operatorname{Fix}\left(g_{x}\right)=\operatorname{Fix}\left(\Phi\left(g_{x}\right)\right.\right.\right. \\
=\left(\mathcal{O}_{2}-\operatorname{int}\left(\operatorname{supp}\left(\Phi\left(g_{x}\right)\right)\right) \cup\{\bar{h}(x)\}\right.
\end{gathered}
$$


SO

$$
\bar{h}\left(\mathcal{O}_{1}-\operatorname{int}\left(\operatorname{supp}\left(g_{x}\right)\right)=\mathcal{O}_{2}-\operatorname{int}\left(\operatorname{supp}\left(\Phi\left(g_{x}\right)\right)\right.\right.
$$

and therefore

$$
\bar{h}\left(\operatorname{int}\left(\operatorname{supp}\left(g_{x}\right)\right)=\operatorname{int}\left(\operatorname{supp}\left(\Phi\left(g_{x}\right)\right)\right.\right.
$$

and so $\bar{h}$ maps basic open sets to basic open sets and so $\bar{h}$ is continuous.

Similarly, $\overline{h^{-1}}$ is continuous. Note that by construction

$$
\bar{h} \circ \overline{h^{-1}}=\operatorname{Id} \text { on } \mathcal{O}_{2}-\Sigma_{2}
$$

and

$$
\overline{h^{-1}} \circ \bar{h}=\operatorname{Id} \text { on } \mathcal{O}_{1}-\Sigma_{1} .
$$

Since $\mathcal{O}_{2}-\Sigma_{2}$ is dense in $\mathcal{O}_{2}$ and $\mathcal{O}_{1}-\Sigma_{1}$ is dense in $\mathcal{O}_{1}$, we have that $\bar{h} \circ \overline{h^{-1}}=\operatorname{Id}$ on $\mathcal{O}_{2}$ and $\overline{h^{-1}} \circ \bar{h}=\operatorname{Id}$ on $\mathcal{O}_{1}$. Hence $\overline{h^{-1}}=(\bar{h})^{-1}$ and so $\bar{h}$ is a homeomorphism that induces the group isomorphism $\Phi$. The proof of the main part of Theorem Ais complete.

\section{Proof of Theorem B}

To prove Theorem B, given a group isomorphism $\tilde{\Phi}: \operatorname{Diff}_{\text {Orb }}^{r}\left(\mathcal{O}_{1}\right) \rightarrow$ $\operatorname{Diff}_{\text {Orb }}^{r}\left(\mathcal{O}_{2}\right)$, we first show that $\tilde{\Phi}$ induces a group isomorphism $\Phi$ : $\operatorname{Diff}_{\text {red }}^{r}\left(\mathcal{O}_{1}\right) \rightarrow \operatorname{Diff}_{\text {red }}^{r}\left(\mathcal{O}_{2}\right)$. Let $\tau: \operatorname{Diff}_{\text {Orb }}^{r}(\mathcal{O}) \rightarrow \operatorname{Diff}_{\text {red }}^{r}(\mathcal{O})$ denote the forgetful homomorphism. Define $\Phi$ by $\Phi(f)=\tau \circ \tilde{\Phi} \circ \tau^{-1}$. We show that this map is well-defined. Suppose $\tilde{f}_{1}, \tilde{f}_{2} \in \tau^{-1}(f)$. Note that $\left.\tilde{f}_{1}\right|_{\mathcal{O}_{1}-\Sigma_{1}}=$ $\left.\tilde{f}_{2}\right|_{\mathcal{O}_{1}-\Sigma_{1}}$. If we consider the manifolds $\mathcal{O}_{i}-\Sigma_{i}$, the groups of diffeomorphisms $\left.\operatorname{Diff}{ }_{\text {Orb }}^{r}\left(\mathcal{O}_{i}\right)\right|_{\mathcal{O}_{i}-\Sigma_{i}}$, (the restrictions of elements of Diff ${ }_{\text {Orb }}^{r}\left(\mathcal{O}_{i}\right)$ to $\mathcal{O}_{i}-\Sigma_{i}$ ), and the group isomorphism $\tilde{\Phi}$ between them, the hypotheses of Rubin's theorem are satisfied (by Lemma 26), and thus there exists a homeomorphism $h: \mathcal{O}_{1}-\Sigma_{1} \rightarrow \mathcal{O}_{2}-\Sigma_{2} \operatorname{with}_{\tilde{\Phi}} \tilde{\Phi}(\tilde{f})=h \circ \tilde{f} \circ h^{-1}$ for all $\left.\tilde{f} \in \operatorname{Diff}{ }_{\text {Orb }}^{r}\left(\mathcal{O}_{1}\right)\right|_{\mathcal{O}_{1}-\Sigma_{1}}$. We then conclude that $\tilde{\Phi}\left(\tilde{f}_{1}\right)=\tilde{\Phi}\left(\tilde{f}_{2}\right)$ on $\mathcal{O}_{2}-\Sigma_{2}$. This is enough to conclude that $\tau \circ \tilde{\Phi}\left(\tilde{f}_{1}\right)=\tau \circ \tilde{\Phi}\left(\tilde{f}_{2}\right)$ as elements of Diff red $\left(\mathcal{O}_{2}\right)$.

By Theorem A, there is a homeomorphism $h: X_{\mathcal{O}_{1}} \rightarrow X_{\mathcal{O}_{2}}$ inducing $\Phi$. The remainder of the proof is to show that $h$ has local lifts in neighborhoods of each $x \in \Sigma_{1}$ that are $\Gamma_{x}$ equivariant once there are the appropriate homomorphisms between $x$ and $h(x)$. To show that $h$ has the appropriate local lifting properties we examine the group $S(\mathcal{O})=\operatorname{ker}\left(\tau: \operatorname{Diff}_{\text {Orb }}^{r}(\mathcal{O}) \rightarrow \operatorname{Diff}_{\text {red }}^{r}(\mathcal{O})\right)$. Note that it is enough to examine those automorphisms that cover the identity. The previous shows that since $\Phi\left(\operatorname{Diff}_{\text {red }}^{r}\left(\mathcal{O}_{1}\right)\right)=\operatorname{Diff}_{\text {red }}^{r}\left(\mathcal{O}_{2}\right)$, it follows 
that $\tilde{\Phi}\left(S\left(\mathcal{O}_{1}\right)\right)=S\left(\mathcal{O}_{2}\right)$. The homomorphism $\tilde{\Phi}$ induces a local homomorphism $\Theta_{x}: \Gamma_{x} \rightarrow \Gamma_{h(x)}$ of isotropy groups as follows. Let $\left(\tilde{U}_{x}, \Gamma_{x}, \rho_{x}, \phi_{x}\right)$ be an orbifold chart around $x \in \Sigma_{1}, y=h(x)$ and $\left(\tilde{U}_{y}, \Gamma_{y}, \rho_{y}, \phi_{y}\right)$ an orbifold chart around $y$ so that

$$
h\left(\phi_{x}\left(\tilde{U}_{x} / \Gamma_{x}\right)\right)=\phi_{y}\left(\tilde{U}_{y} / \Gamma_{y}\right)
$$

Let $D_{x} \subset \tilde{U}_{x}$ and $D_{y} \subset \tilde{U}_{y}$ be Dirichlet domains for the actions of $\Gamma_{x}$ and $\Gamma_{y}$ respectively. For $z \in D_{x}$ there is a unique $\gamma \in \Gamma_{x}$ so that $\gamma^{-1} \cdot z \in D_{x}$. Let $\sigma_{\gamma} \in S\left(\mathcal{O}_{1}\right)$ be the element that sends $\tilde{U}_{x} \ni z \rightarrow \gamma \cdot z$ (See Proposition 28). Define $\Theta_{x}: \Gamma_{x} \rightarrow \Gamma_{y}$ by $\Theta_{x}(\gamma)=\gamma$ where $\tilde{\Phi}\left(\sigma_{\gamma}\right)=\sigma_{\gamma^{\prime}} \in S\left(\mathcal{O}_{2}\right)$. It is easily checked that this is a group homomorphism. For any $z \in \tilde{U}_{x}$, we define a $\Theta_{x}$ equivariant lift $\bar{h}$ of $h$ by first defining it for $z \in D_{x}$ as the unique $\bar{h}(z)=z \in D_{y}$ so that

$$
\phi_{y}\left(\Gamma_{y} \cdot z\right)=h\left(\phi_{x}\left(\Gamma_{x} \cdot z\right)\right)
$$

and then extending to $z \in \gamma \cdot D_{x}$ by letting $\bar{h}(z)=z$ where

$$
\Theta_{x}\left(\gamma^{-1}\right) \cdot z \in D_{y}
$$

and

$$
\phi_{y}\left(\Gamma_{y} \cdot z\right)=h\left(\phi_{x}\left(\Gamma_{x} \cdot z\right)\right)
$$

By construction, this is a $\Theta_{x}$ equivariant lift of $h$ to a neighborhood of $x$ and so the triple $\left(h, \Theta_{x}, \bar{h}\right)$ is an (unreduced) orbifold homeomorphism inducing the group isomorphism $\tilde{\Phi}$.

\section{Proof of Theorem C}

We sketch the proof of this result. Complete proofs may be found in Borzellino and Brunsden. ${ }^{13,12}$ The general idea is to mimic the details as much as possible of the analogous proof for manifolds. That this is possible leads to some extensions of several results on group actions.

It suffices to construct a neighborhood of the identity homeomorphic to some open set of the appropriate topological vector space (a Banach space for $1 \leq r<\infty$ and a Fréchet space for $r=\infty$ ). Let $\mathcal{N O}$ be the normal orbibundle of the diagonal $\Delta(\mathcal{O})$ in $\mathcal{O} \times \mathcal{O}$. That is, it is locally the quotient of $T_{\Delta(\mathcal{O})}(\mathcal{O} \times \mathcal{O}) / \tilde{T} \Delta(\mathcal{O})$, i.e. the normal orbibundle of the diagonal in the Cartesian product $\mathcal{O} \times \mathcal{O}$. There is an orbifold tubular neighborhood of $\Delta(\mathcal{O})$ 
that is covered by $\mathcal{N O}$. With a little work, one can see that $\mathcal{N O}$ is isomorphic to $T \mathcal{O}$. We let

$$
\overline{\exp }: \mathcal{N O} \rightarrow \mathcal{O} \times \mathcal{O}
$$

be the exponential map induced by a Riemannian metric on $\mathcal{O}$. This induces a map which by abuse of notation we also call $\overline{\exp }$,

$$
\overline{\exp }: D_{\mathcal{O}}^{r}(\mathcal{O}) \rightarrow C_{\text {Orb }}^{r}(\mathcal{O}, \mathcal{O})
$$

Where $D_{\mathcal{O}}^{r}(\mathcal{O})$ is the topological vector space of $C^{r}$ orbisections of the orbibundle $\mathcal{N O}$. The above defines a $C^{0}$ neighborhood of the identity (which corresponds to $\overline{\exp }(0))$ as in the manifold case. A sufficiently small $C^{1}$ neighborhood of the identity in $C_{\text {Orb }}^{r}(\mathcal{O}, \mathcal{O})$ is in $\operatorname{Diff}_{\text {Orb }}^{r}(\mathcal{O})$ and so this gives $\operatorname{Diff}_{\text {Orb }}^{r}(\mathcal{O})$ a manifold structure. For details, see Borzellino and Brunsden. ${ }^{13}$

\section{Acknowledgements}

The authors would like to thank the organizers and Howard University for their generosity and hospitality. We also wish to thank the other participants for a most interesting and stimulating conference

\section{References}

1. M. Anderson and J. Cheeger, Diffeomorphism finiteness for manifolds with Ricci curvature and $L^{n / 2}{ }_{\text {-norm }}$ of curvature bounded, Geom. Funct. Anal. 1, no. 3, 231-252 (1991)

2. A. Banyaga, On Isomorphic Classical Diffeomorphism Groups I, Proc. Amer. Math. Soc. 98, 113-118 (1986)

3. A. Banyaga, On Isomorphic Classical Diffeomorphism Groups II, J. Diff. Geometry 28, 23-25 (1988)

4. A. Banyaga, The structure of classical diffeomorphism groups, Mathematics and its Applications, vol. 400, Kluwer Academic Publishers, Dordrecht, 1997.

5. A. Banyaga, Isomorphisms Between Classical Diffeomorphism Groups, CRM Proceedings and Lecture Notes, 15 (1998), 1-15.

6. A. Banyaga, Sur la Structure du Groupe des Difféomorphismes qui Préservent une Forme Symplectique, Comment. Math. Helv. 53, no. 2, 174-227 (1998)

7. J. Borzellino, Riemannian Geometry of Orbifolds, PhD. Thesis, Univ. Calif. Los Angeles, Spring 1992. 
8. J. Borzellino. Orbifolds of Maximal Diameter, Indiana U. Math. J. 42, 37-53 (1993)

9. J. Borzellino. Orbifolds With Lower Ricci Curvature Bounds, Proc. American Mathematical Society 125, no. 10, 3011-3018 (1997)

10. J. Borzellino and V. Brunsden, Determination of the topological structure of an orbifold by its group of orbifold diffeomorphisms, Preprint (1999).

11. J. Borzellino and V. Brunsden, An Automorphism of the Orbifold Diffeomorphism Group which is not an Inner Automorphism, Preprint (2000).

12. J. Borzellino and V. Brunsden, A Differential Topologist's Toolkit for Orbifolds, In preparation (2002).

13. J. Borzellino and V. Brunsden, On the Topology of the Group of Orbifold Diffeomorphisms of a Smooth Orbifold, In preparation (2002).

14. J. Borzellino and S. Zhu. The Splitting Theorem for Orbifolds, Illinois J. Math. 38, 679-691 (1994)

15. G. Bredon, Introduction to the Theory of Transformation Groups, Academic Press, New York 1972.

16. V. Brunsden, Local Rigidity and Group Cohomology I: Stowe's Theorem for Banach Manifolds, Bull. Aus. Math. Soc. 59, no. 2, 271-295 (1999)

17. V. Brunsden, Local Rigidity and Group Cohomology II: Anosov-like Actions, preprint (2000).

18. A. Dress, Newman's Theorems on Transformation Groups, Topology 8 , 203-207 (1969)

19. R. Filipkiewicz, Isomorphisms between diffeomorphism groups, Ergodic Theory Dynam. Systems 2, 159-171 (1982)

20. K. Fukaya and K. Ono, Arnold conjecture and Gromov-Witten invariant, Topology 38, no. 5, 933-1048 (1999)

21. M. Hirsch, Differential Topology, Springer-Verlag, New York, 1976.

22. M. Rubin, On the reconstruction of topological spaces from their homeomorphism groups, Trans. Amer. Math. Soc. 312, no. 2, 437-538 (1989)

23. T. Rybicki, Isomorphisms between groups of diffeomorphisms, Proc. Amer. Math. Soc. 123, no. 1, 303-310 (1995)

24. I. Satake. On a Generalization of the Notion of Manifold, Proc. Nat. Acad. Sci. USA 42, 359-363 (1956)

25. I. Satake. The Gauss-Bonnet Theorem for V-Manifolds, Jour. Math. Soc. Japan 9, 464-492 (1957)

26. P. Scott. The Geometries of 3-manifolds, Bull. London Math Soc. 15, 401-487 (1983)

27. W. Thurston, The Geometry and Topology of 3-Manifolds, Lecture notes, Princeton University Mathematics Department, (1978). 